\title{
The energy-efficient MDA-SMAC protocol for wireless sensor networks
}

Ke Wang ${ }^{1,3}$, Xiaohu Zhao ${ }^{2^{*}}$, Yao Shi ${ }^{1}$, Donghong $X u^{1,3}$ and Ruoyu $\mathrm{Li}^{1}$

\begin{abstract}
Nodes in original S-MAC protocol just can visit the channel in the scheduling and listening stage. The working schema may result in data latency and high conflict. To solve those above problems, we split scheduling duty into multiple micro-duties. By using different micro-dispersed contention channel, the sensor nodes reduce the collision probability of the data. Aiming at detecting the fixed duty cycle in S-MAC protocol, on the basis of the micro-duty and buffer queue length, this paper presents an adaptive duty cycle and back-off algorithm. While using different back-off algorithm with different duty cycles, sensor node Fast-Binary Exponential Backoff and Conflict-Avoid-Binary exponential Backoff algorithm separated are applied to reduce data latency further reduce the conflict probability. Combining both of the improvements, we propose a modified S-MAC protocol. Comparing the performance of SMAC protocol and Division-Multiple Access-Media Access Control (MDA-SMAC) protocol on the NS-2 simulation platform, the results show that MDA-SMAC protocol performs better in terms of energy consumption, latency, and effective throughput than S-MAC protocol
\end{abstract}

Keywords: Wireless sensor networks, S-MAC protocol, Micro-duty, MDA-SMAC protocol

\section{Introduction}

Wireless sensor networks are consisting of thousands of extremely small and cheap devices that can sense the environment and communicate the data as required. Wireless sensor networks have emerged as one of the first real applications of ubiquitous computing. So saving energy of sensor nodes is the important issue when designing wireless sensor network applications. Media Access Control (MAC) protocol, which determines how sensors get access to the channel, directly controls the communication module with most energy consumption of the nodes. The design of energy-efficient Division-Multiple Access- Media Access Control (MDA-SMAC) protocol is one of the hot researches about wireless sensor networks.

Currently, many researchers of MAC protocol for wireless sensor networks conducted in-depth research in this field and also achieved some results [1]. There is no unity criterion for dividing MAC protocol into different classes in wireless sensor networks: according to the number of occupied channels, it can be divided into multi-channel

\footnotetext{
* Correspondence: wklx041030@163.com

${ }^{2}$ The National Joint Engineering Laboratory of Internet Applied Technology of Mines, Xuzhou, China

Full list of author information is available at the end of the article
}

and single-channel MAC protocol; according to data communication types, it can be divided into unicast and multicast MAC protocol; according to the variability of nodes transmission power, it can also be divided into fixed and variable power MAC protocol. Based on the node access channel method, the most wildly used classification divides the MAC protocol into three classifications: MAC protocol based on channel allocation mechanism, hybrid MAC protocol, and MAC protocol. MAC protocol based on competition mechanism for wireless sensor networks has the following features: better adaption to network topology changes, no centralized control of the large number of scattered sensor nodes, and that channel is only in use when data transmission occurs. Otherwise, it turns into sleep or low-power state, dramatically reducing the energy consumption.

Due to its inherent flexibility, scalability, and the advantage of tackling the problem of data fluctuation, competition-based MAC protocol is widely used with high research value. Sensor-Media Access Control (S-MAC) protocol [2], proposed by the USC/ISI Wei Ye, John Hediemann et al, is a typical completion-based protocol in wireless sensor networks. Almost all other competitionbased MAC protocols borrowed from some of the thinking 
of this protocol or improved on its basis. This IEEE802.11 MAC-based protocol, especially proposed for solving the problem of limited energy storage of sensor nodes in wireless sensor network, achieves a better energy efficiency effect, with assumptions that the network can tolerate a certain delay, and allowance for node sleep periodically. Since the S-MAC protocol uses a competitive mechanism for accessing channel, it also has good scalability. The main mechanism applied in the S-MAC protocol are timing synchronization, flow-adaptive listening, crosstalk avoid, and fragment messages transmit.

In the S-MAC protocol, nodes use a locked duty cycle to work, which cannot get with the changes of network traffic. Sleep listen scheduling schema for nodes are fixed: at the beginning of each listen/sleep duty, nodes compete for the channel, with nodes within a cluster working in a synchronized way. This approach leads to a less node working hours, long sleep time, wasting a lot of time resources, and large network latency. During the stage of listening, all nodes try to get access to channel. So when network traffic flow is large or in a dense Wireless Sensor Net (WSN) environment, the conflict between the nodes will exacerbate, which led to multiple nodes retransmission back-off.

For solving the above mentioned problem, we split scheduling duty into multiple micro-duties. By using different micro-dispersed contention channel, the sensor nodes reduce the collision probability of the data. Aiming at detecting the fixed duty cycle in S-MAC protocol, on the basis of the micro-duty and buffer queue length, this paper presents an adaptive duty cycle and back-off algorithm. While using different back-off algorithm with different duty cycles, sensor node Fast-Binary Exponential Backoff (F-BEB) and Conflict-Avoid-Binary exponential Backoff (CA-BEB) algorithm separated are applied to reduce data latency further reduce the conflict probability.

\section{Method}

\subsection{Micro-duty method}

\subsubsection{Basic principles of micro-duty}

Both S-MAC and Time-Media Access Control (T-MAC) protocol adopt conflict resolution ways of IEEE802.11MAC when conflict occurs among nodes: use a kind of back-off algorithm to postpone node access to the channel.

S-MAC has three major components: periodic listen and sleep, collision and overhearing avoidance, and message passing.

Each node goes to sleep for some time and then wakes up and listens to see if any other node wants to talk to it. During the 2013 3rd IEEE International Advance Computing Conference (IACC) 489 sleep, the node turns off its radio and sets a timer to awake it later. The duration of time for listening and sleeping can be selected according to different application scenarios. For simplicity, these values are the same for all the nodes. Network and application assumptions are the node to node communication; in network processing (like data aggregation), application have long idle period and can tolerate some latency. Periodic listen and sleep is used to avoid idle listening. Contention mechanism is the same as in IEEE 802.11 to avoid collision. Overhearing is avoided by letting interfering nodes go to sleep when they hear RTS/CTS packets. So that they may not hear the long data packet, the ACK.NAV value is used for this purpose. Message passing is used to reduce control overhead in contrast with 802.11. Long messages are broken into small fragments and transmitted as a burst. Extra delay was because of node periodic sleeping. Adaptive listening has also been introduced to improve the latency in multi-hop scenarios (Fig. 1).

Combining those typical back-off algorithms, we can draw their general formula below:

$$
W_{i+1}=\left\{\begin{array}{c}
\min \left(m_{i} \times C W_{i}, C W_{\max }\right) \\
\max \left(r_{i} \times C W_{i}, C W_{\text {min }}\right) \\
\min \left(C W_{i}+l_{i}, C W_{\text {max }}\right)
\end{array}\right.
$$

Formula 2-1 means the current window value are changed according to the accessing state of nodes in back-off algorithms. We propose a theoretical analysis of these algorithms based on these algorithms. Suppose there is a current node in the network, each node will definitely send data after a period of channel competition. We set $W(t)$ as the back-off counter size at

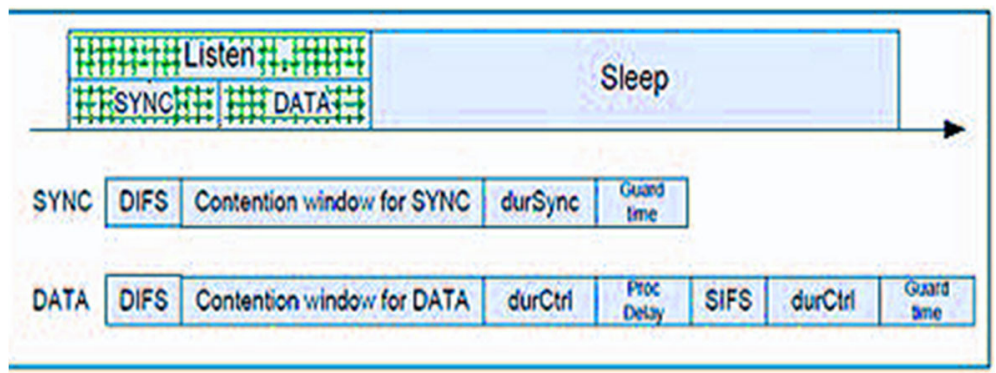

Fig. 1 The design of SMAC protocol 
time $t$ (a discrete-time set here), $t$ and $t+1$ are two consecutive time slots, and $T$ is the size of a time slot. After the node selected $W(t)$, it is clear that the back-off window value of the node is knowable during the period of $W(t)$ retreating to 0 ; therefore, a Markov chain model could not be constructed. We set another stochastic process $R(t)=k$ to indicate the number of node collision times at slot time $t$, and we can get the formula 2-2 to 2-5, where $p$ denotes the possible collision probability of data transmission in the channel and $k$ denotes the frequency of node accessing channel.

$$
\mathrm{P}\{j, k \mid j, k+1\}=1
$$

Equation 2-2 means for each time the node enters into a new time slot (i.e., channel being listened to be idle); its back-off timer value is decreased by 2 .

$$
\mathrm{P}\{0, k \mid j, 0\}=\frac{1-p}{W_{0}}
$$

Equation 2-3: after the packet transmission, the number of its current collision time is set to be zero, and the back-off window value will be re-selected.

Equation 2-4: if the node collision occurs, the number of collision will be added by 1 , and the back-off window value will be re-selected.

$$
\mathrm{P}\{i, k \mid j-1,0\}=\frac{p}{W_{i}}
$$

Equation 2-5: once the collision time reaches the up limit, the node will reset the contention window.

$$
\mathrm{P}\{n, k \mid m, 0\}=\frac{p}{W_{n}}
$$

According to the formula 2-2 to 2-5, limiting distribution can be expressed as:

$$
Q_{i, k}=\lim _{t \rightarrow \infty} p\{W(t)=j, R(t)=k\}
$$

According to Kolmogorov-Chapman (KC) equation, we draw the following conclusion:

$$
Q_{j, 0}=p^{j} \cdot Q_{0,0} \mathbf{j} \in(0, m)
$$

By Equation 2-8, the system can derive the steady state conditions of the collision probability of the node:

$$
Q_{0,0}=\frac{2(1-2 p)(1-p)}{(1-2 p)(W+1)+p W\left[1-(2 P)^{m}\right]}
$$

On the basis of this formula, we can get the following equation.

$$
\begin{gathered}
\tau=\sum_{i=0}^{m} Q_{i, 0}=\frac{1}{1-p} \cdot Q_{0,0} \\
=\frac{2(1-2 p)}{(1-2 p)(W+1)+p W\left[1-(2 p)^{m}\right]}
\end{gathered}
$$

This formula express the relationship between the collision probability. The node can be drawn from the condition probability as shown below:

$$
p=1-(1-\tau)^{n-1}
$$

Literature [3] solves this kind of problem by applying another Markov chain model, deriving the formula (2-11).

$$
n=1+\frac{\log _{2}(1-p)}{\log \left\{1-2(1-2 p) /\left\{(1-2 p)(W+1)+p W\left[1-(2 p)^{m}\right]\right\}\right\}}
$$

$$
(2-11)
$$

As can be seen, the probability of data transmission depends on the collision probability $p$, which has a close link with the number of nodes in the current conflict region.

Through the above two analytical methods, we can get the following result. When the network load is light, less number of nodes will visit the channel less and less conflicts will occur among nodes. When the network load is heavy, a large number of nodes will compete for the channel, more conflicts will be aroused within the same time, and more energy will be consumed.

S-MAC protocol is thoroughly studied in Literature [4]. The numbers of nodes in the network are unchanged by man, but the probability of conflict between the nodes and energy consumption can also be reduced by controlling the arrival time of node for the channel competition, which means different nodes visit channel in different time phrases Hence we propose the following improved idea. A mechanism is introduced, by which, virtual nodes within a cluster of S-MAC protocol dispersedly compete for the channel. Part of the nodes listen the channel when the other nodes are in a sleep state, while shift into a sleep state while other nodes listens the channel. In this manner, the nodes are dispersed by different time intervals to contend for the channel, reducing the probability of collision. Meanwhile in a large duty, nodes and data communications can be carried out repeatedly in a manner similarly to Time-Division-Multiple Access (TDMA), thereby reduces data latency.

In order to achieve its design objective of decentralized nodes and multi-period multi-node communication within the mission ring, this paper presents a new scheduling duty: the micro-duty. Nodes in S-MAC protocol, with the same scheduling period formed a virtual cluster, consisting of multiple virtual clusters throughout the network. Similarly, micro-clusters a kind of cluster is smaller than virtual cluster in S-MAC protocol. Setting micro-duty as the synchronous scheduling unit for each 
node, nodes working in the same micro-duty compose the smaller micro-clusters, and micro-clusters constitute the virtual clusters in S-MAC.

The number of micro-clusters is equal to the number of micro-duty. Only nodes working in the same microduty only belong to the same micro-cluster. One scheduling duty in S-MAC protocol can be split into $n$ microduties; therefore, the number of micro-clusters is also $n$.

\subsubsection{The component of micro-duties}

In S-MAC protocol, three stages of nodes:

synchronization, listening, and sleeping constitute a complete scheduling period, that is, a task loop (microduty). Within each task loop, nodes firstly synchronize and then listen and send the data, and shift to sleeping mode when sleep clock arrives, as shown in Fig. 2.

Division of micro-duty: the listener/sleep stage of a task loop in the original S-MAC protocol is divided into multiple independent continuous listening shorter sleep stages. For node, data are transmitted at each micro- listener stage, and then turn into the sleep state to conserve energy at each micro-sleep stage. Multiple successive micro-duties constitute the micro listener/sleep duty of the original S-MAC protocol as shown in Fig. 3.

\subsubsection{Choosing and setting state}

In MDA-SMAC protocol, nodes first need to determine the number of their own micro-duty and status of each micro-duty, and then adjust its duty cycle according to network traffic micro-duty. So, how to choose the number of micro-node duty they own, and set the status of each micro-duty for themselves, is the primary task during nodes initialization.

Conserving energy for nodes is the primary task of wireless sensor network, data transmission are permitted to have a certain delay on the condition that energy are ensured to be efficiently conserved. To solve this problem of energy-constrain, Literature $[5,6]$ proposed a clustering protocol based on residual energy. Therefore, on the basis of this paper, we propose an approach to select the number of micro-duty based on the remaining energy, and set the state of the micro-duty.

With $10 \%$ duty cycle, the task loop is divided into 10 micro-duties. The initial energy of the node is $E_{\text {init }}$, after a period of operation, its residual energy is $E_{\text {residual }}$. Hence the number of micro-duties of the node can be deducted according to formula 4-24, wherein N represents the number of the divided micro-duty.

$$
n=\left\{\begin{array}{cl}
1 & \frac{E_{\text {residual }}}{E_{\text {init }}} \times N \leq 1 \\
\left\lceil\frac{E_{\text {residual }}}{E_{\text {init }}} \times N\right] & \frac{E_{\text {residual }}}{E_{\text {init }}} \times N>1
\end{array}\right.
$$

Node is calculated according to the formula 2-12 a number of micro-duty of its own is $\mathrm{n}$, then the node is set to work only the first $\mathrm{n}$ state in 10 micro-duty, the rest of the micro-duty in a sleep state.

The smaller the residual energy of nodes, the fewer the number of its access channel. If the node is the initial energy of $1000 \mathrm{~J}$, when the remaining energy is less than $100 \mathrm{~J}$, the remaining energy will be the case with the initial energy ratio is less than 1, there is necessary to perform further calculations, then the node is calculated according to the formula 2-13:

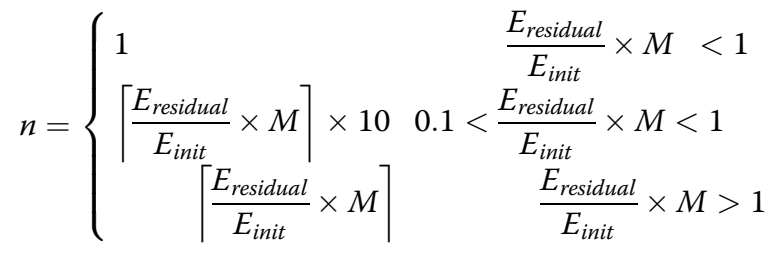

After completing the number selection of microduty, nodes need to set the status of each micro-duty. First, the node choose $m$ random numbers from $\{1,2$, $3, \ldots m\}$; each random number are used to represent each micro-duty of a node. Node status in each micro-duty is determined by the following rules: when the state of the random number is greater than or equal to the threshold value, the node is in listening state within the micro-duty; when the random number is less than the threshold value, the node is in sleeping state within the micro-duty. Threshold is also need to be set by considering the residual energy of the node. This paper combines the threshold value with the owing number of micro-duties of node. The threshold is set according to the formula 2-14:

$$
F=\lceil n / 2\rceil
$$

By setting a threshold in the formula 2-14, it is feasible that node can listen to the channel within half of the

\begin{tabular}{|l|l|l|} 
& \multicolumn{1}{c|}{ listen } \\
\cline { 2 - 3 } & For SYNC & sleep \\
\hline
\end{tabular}

Fig. 2 The schedule of S-MAC 


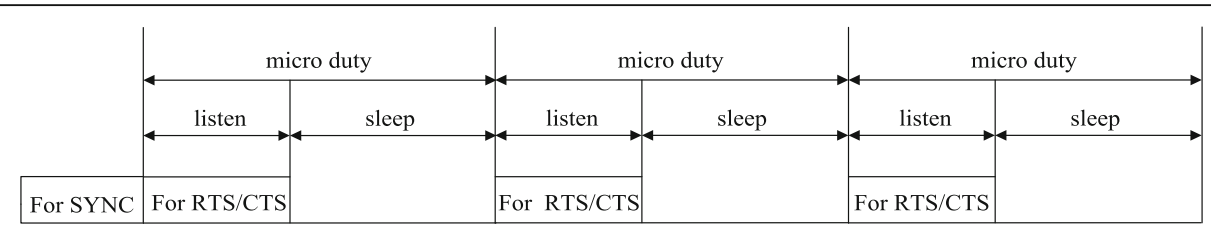

Fig. 3 The micro-duty

micro-duty and sleep within the other half micro-duty. In this way, the nodes extend the sleep time, and further reduce the energy consumption.

By setting the state of micro-duty in this method, the node can use a plurality of micro-duties for communication, to get the goal of multiple nodes communicating over a large period of the duty. And due to the random selection, nodes are dispersed in different micro-duty. Number of nodes that compete in each micro-duty is less than the number of nodes of contention channel in S-MAC protocol (all nodes of the virtual cluster) less. Hence, collision probability of the data is decreased, and then, more energy is saved. The threshold value determines whether the node should get access to channel within the microduty. For a single node, no access to channel means the node sleep time is longer, lower energy consumption, and longer network lifetime.

\subsection{Suitable method for traffic duty cycle}

In this paper, length of buffer queue is adopted as a key factor for assessing network traffic, and then, the duty cycle of MDA-SMAC protocol is dynamically changed. Defining rules in the MDA-SMAC protocol as follows: if the buffer queue length is greater than a certain threshold value, then the duty cycle of the node should be increased due to more packets need to be transmitted and more crowded network traffic.

After many experiments, we consider that if the buffer queue length is over 60 , the current network traffic is large, and nodes need to expand the duty cycle. Therefore, we use 60 (a buffer length) as the duty cycle threshold.

Literature $[7,8]$ also proposed dynamic duty strategy on basis of S-MAC protocol. They further study the dynamic duty cycle mechanisms in these protocols, where the node still compete in a unified listening period for channel, causing larger conflict probabilities between nodes. In the MDA-SMAC protocol, nodes use micro-cycle to schedule and work is distributed within the micro-duty, so the probability of conflict between the nodes is significantly reduced. Therefore, adaptive duty cycle in MDA-SMAC protocol means adjusting the duty cycle of the micro-duty.
In the MDA-SMAC protocol, nodes use formula 2-15 to adjust the duty cycle of micro-duty.

$$
\text { DCnew }=\left\{\begin{array}{cc}
\min \left(2 \times D C_{\text {old }}, D C_{\max }\right) & Q_{\text {length }} \geq 60 \\
D C_{\text {old }} & Q_{\text {length }}<60
\end{array}\right.
$$

\subsection{BEB back-off algorithm}

S-MAC protocol use BEB back-off [9] algorithm regardless of the current network traffic condition. BEB algorithm stipulates different upper limit for retransmission. In general, the upper limit of the retransmission of the node in BEB algorithm is 7, and the corresponding range for the back-off window size is $[0,127]$. If the RTS/CTS mechanism are adopted, the upper limit thereof is 4, corresponding to the backoff window value interval $[0,16]$; NS2 [10] [11] in the S-MAC protocol set the maximum back-off window to be 63. However, fixed setting method for window back-off interval is not suitable for dynamically changing wireless sensor networks, and therefore, developing the flow-adaptive back-off algorithm is a direction for improving S-MAC protocol.

All back-off algorithms contain two fields: $C W_{\min }$ and $C W_{\max }$, representing minimum back-off window value and maximum back-off window value, respectively. Back-off window value is randomly selected from two. Therefore, one of the key point for flowadaptive back-off algorithm is dynamically changing the size of $C W_{\min }$ and $C W_{\max }$. On this basis, we propose two improved BEB algorithm: F-BEB and CA-BEB.

\subsubsection{F-BEB algorithm}

This paper sets a threshold $L_{\text {rate }}$ for buffer utilization rate. The threshold parameter tuning in terms of buffer space and inclusion of traffic load in congestion notification along with average size is used

After several experiments, we find that when the buffer queues length becomes less than 12 that indicates lower network traffic. So set $L_{\text {rate }}$ to be $12 \%$. The back-off window range is modified according to formula 2-16 and 2-17: 


$$
\mathrm{CW}_{\text {newmin }}=\left\lceil\left(1+\text { Buffer }_{\text {rate }}\right) / 2 \times \mathrm{CW}_{\min }\right\rceil
$$

$$
\mathrm{CW}_{\text {newmax }}=\left\lceil\left(1+\text { Buffer }_{\text {rate }}\right) / 2 \times C W_{n}\right\rceil
$$

The lower the utilization rate, the less data will need to be send. Associating the back-off window interval of standard duty cycle of nodes with the current buffer utilization rate, back-off window range of the node can be dynamically reduced [12-15]. For each time, small value of the back-off window size selection leads to less back-off time of the node, allowing fast accessing to channels. Therefore, under the standard duty cycle, if the buffer utilization rate is less than $L_{\text {rate }}$, then the node uses F-BEB algorithm for channel competition. F-BEB back-off algorithm is described as [12]. On the whole, FBEB algorithm applies three strategies: freezing the counter in the busy period of the channel, the back-off window value increases.

\subsubsection{CA-BEB algorithm}

First, the node will alter its back-off window range according to the formula 2-18 and 2-19,

$$
\begin{aligned}
& \mathrm{CW}_{\text {newmin }}=\left\lceil\left(1+\text { Buffer }_{\text {rate }}\right) \times C W_{\text {min }}\right\rceil \\
& \mathrm{CW}_{\text {newmax }}=\left\lceil\left(1+\text { Buffer }_{\text {rate }}\right) \times C W_{\max }\right\rceil
\end{aligned}
$$

In CA-BEB algorithm, nodes change the back-off window interval according to its current buffer utilization, which provides two main benefits, the detailed description of CA-BEB algorithm can be viewed in [13]. In the algorithm, node dynamically changes its back-off window interval according to the current buffer utilization rate, rather than simply doubling the node back-off window interval. Hence, the size of back-off window interval could properly be controlled, preventing oversized window problem for nodes to some extent and taking into account the competing fairness of channel access as well [16-20].

In general, F-BEB algorithm reduces the extent of starvation and improves fairness and CA-BEB more relies on the capability of the stations to detect a collision by hearing their own transmission.

\section{Results and discussion from single-hop simulation}

Experiment uses one hop topology for simulation. In NS2 platform, node positional relationship is shown in Fig. 4. Twenty-one nodes are deployed in the 100 $\mathrm{m} \times 100 \mathrm{~m}$ area; the node 0 is at the network center. Sink nodes are responsible for receiving data, and the other 20 nodes are located around the node 0 sending data to sink.

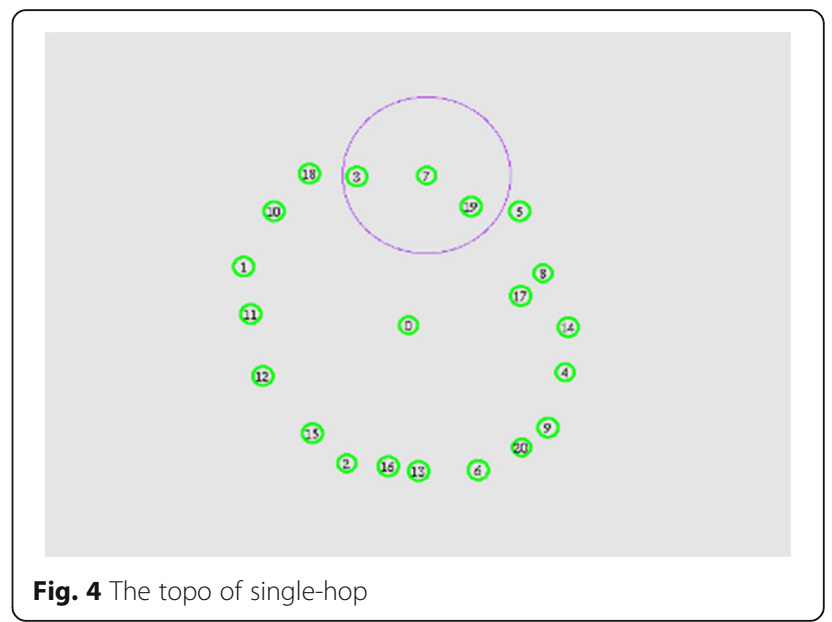

Since MDA-SMAC protocol uses stochastic manner to decide which micro-cycle will be selected for nodes to listen. For each time, stochastic results may be different, so this paper uses the mean value of multiple experiments as simulation results (Table 1 ).

Data is sent from $5 \mathrm{~s}$, continuing to $30 \mathrm{~s}$. In the simulation process, different nodes access channel at different times, and at the same time, by changing packet transmission time intervals, the size of the network traffic is stimulated. The smaller transmission interval, the more node sending packets stimulate larger current network traffic; the larger the transmission interval, the fewer node sending packets stimulate less network traffic. In the simulation process, the data packet transmission interval ranges from 1 to 10s, with each interval under 10 tests, taking 10 times the average for the final results.

In this paper, average energy consumption of nodes for the two protocols during each interval of data sending is compared as benchmarks. Figure 5 shows the comparison of the energy performance of the two protocols. As can be seen from the figure, energy consumed in the use of MDA-SMAC protocol for node sends a

Table 1 Some simulation parameters of experiment 1

\begin{tabular}{cc}
\hline parameters & value \\
\hline Region size & AODV \\
Communication bandwidth $/ \mathrm{kbps}$ & $100 \mathrm{~m} \times 100 \mathrm{~m}$ \\
Sending power consumption $/ \mathrm{mW}$ & 20 \\
Receiving power & 200 \\
consumption $/ \mathrm{mW}$ & 200 \\
Sleeping power consumption $/ \mathrm{uW}$ & 1 \\
Node primary power $/ \mathrm{J}$ & 100 \\
Nodes number & 21 \\
Max length of buffer queue & 50 \\
Communication radius $/ \mathrm{m}$ & 100 \\
\hline
\end{tabular}




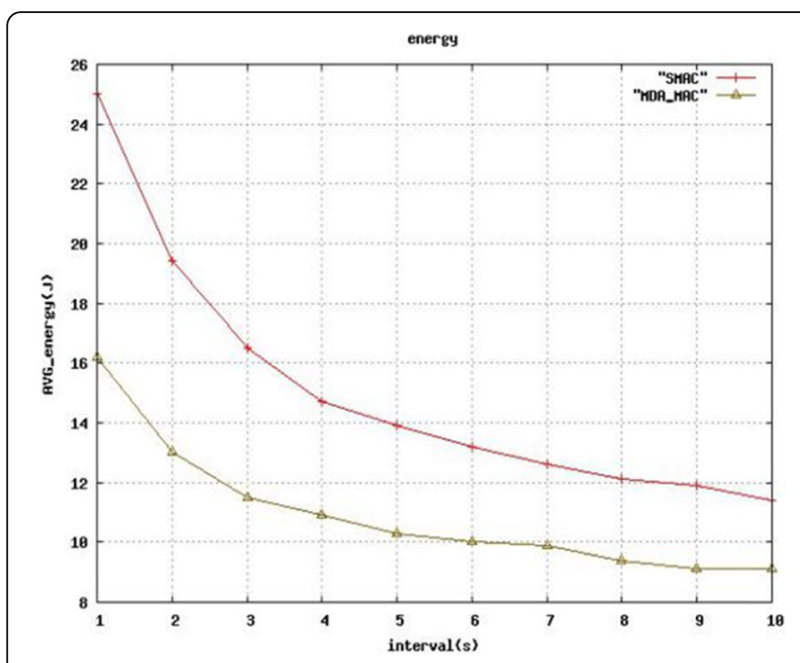

Fig. 5 The comparison of average energy consumption

packet of each transmission interval is less than that of the S-MAC protocol. When the network traffic is large, the conflict probability between the nodes in S-MAC protocol also increases. Nodes may require multiple back-off retransmissions, therefore consuming more energy. The MDA-SMAC dispersed nodes to the microcycle, reducing the probability of conflict, while the use of CA-BEB algorithm at a high flow period further reduces the probability of conflict between the nodes, so the energy consumption is less than that of S-MAC protocol. With the gradual increase of the transmission interval, the network traffic decreases, the energy consumption for the two protocols are reduced. But overall, the energy consumed by MDA-SMAC protocol is less than that of S-MAC protocol.

Figure 6 shows the comparison of mean delay. As can be seen, when the network traffic is high, fixed duty

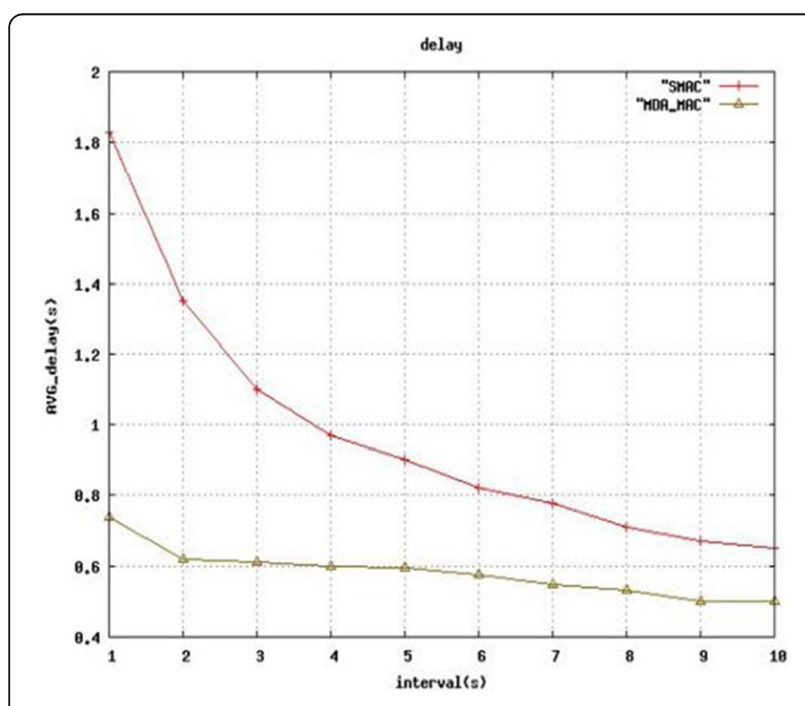

Fig. 6 The comparison of average delay cycle and a high S-MAC protocol conflicts result in higher data delay. MDA-SMAC protocol applies a micro-cycle as a scheduling period, in which a node can transmit data in a plurality of micro-cycles, while the node uses adaptive duty cycle flow mechanisms and CA-BEB algorithm to reduce conflict. Hence, data latency of MDA-SMAC is less than that of S-MAC protocol. With the gradual reduction of network traffic, the data transmission delay in both protocols is decreasing. However, due to the F-BEB algorithm that MDA-SMAC protocol adopted, when the network traffic is low, the data latency is still less than that of SMAC protocol.

As shown in Fig. 7, effective throughput of the two protocols is compared. As can be seen from the figure, when the network traffic is larger, the node S-MAC protocol operation may cause a large amount of data packets aggregating in the buffer. This aggregation problem may result in a higher the probability of collision between the nodes and, therefore, a smaller the effective throughput. In MDA-SMAC protocol, data transmission can be repeated within a scheduling period, and the duty cycle of the micro-cycle can be adjusted according to buffer queue length. Thanks to the adaptive back-off algorithm, the effective throughput of MDA-SMACK is higher than that of S-MAC. When the network traffic is small, there are less the number of packets in the network with small conflict probability between the nodes. So there is little difference in terms of effective throughput for both $\mathrm{S}$ MAC protocol and MDA-SMAC protocol (Table 2).

\section{Results and discussion from multi-hop simulation}

Experiment scenarios for multi-hop simulation are shown in Fig. 8, with 40 nodes located in a $100 \mathrm{~m} \times 100$ $\mathrm{m}$ area. These 40 nodes are located separately in two clusters (in this case, cluster refers to all nodes in in the

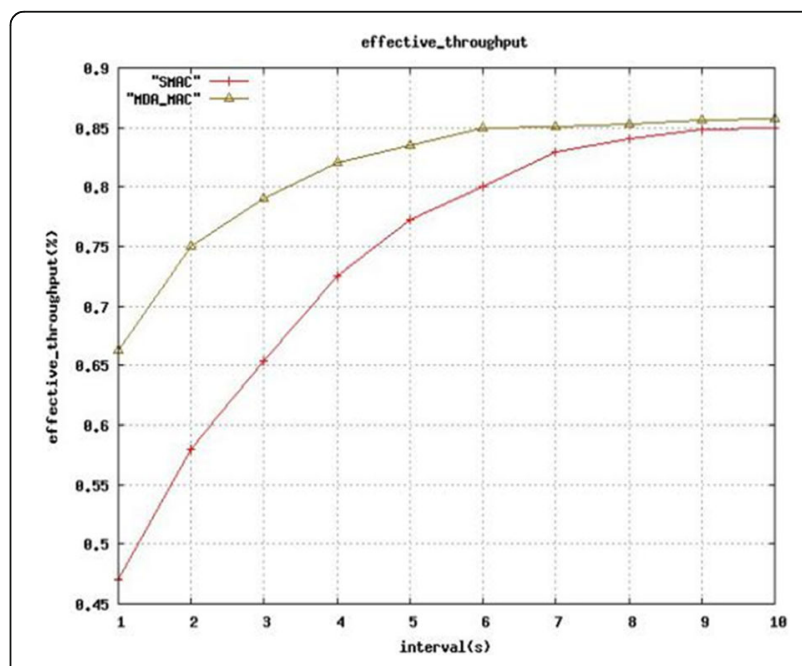

Fig. 7 The comparison of effective throughput 
Table 2 Simulation parameters of experiment 2

\begin{tabular}{cc}
\hline parameters & value \\
\hline Routing protocols for upper layers & AODV \\
Area size & $100 \mathrm{~m} \times 100 \mathrm{~m}$ \\
Communication bandwidth/kbps & 20 \\
Sending power consumption $/ \mathrm{mW}$ & 150 \\
Receiving power & 150 \\
consumption/mW & 1 \\
Sleeping power consumption/uW & 100 \\
Node initial power/J & 41 \\
Number of nodes & 50 \\
Maximum of buffer queue length & 50 \\
\hline Communication radius/m & \\
\hline
\end{tabular}

single-hop range), and the node 0 belongs to two clusters, as the sink node responsible for data transmission.

To ensure data transmission hop-by-hop, the sending power and the receiving power of nodes will be reduced to $150 \mathrm{mw}$, and the communication radius for nodes is $50 \mathrm{~m}$. Experiments are conducted for 10 times under the two-hop environment during each interval of data sending, taking the average of 10 experiments as a simulation result, which takes $50 \mathrm{~s}$ (the nodes within two clusters accessing channel at different times)

In the two-hop scenario, the energy consumption comparison of the two protocols is as shown in Fig. 9. Because of the use of the two-hop scene (more number of nodes than that in experiment one), the conflict probability between the nodes increases. But it can be seen from Fig. 9 that in each contract interval, the energy consumed by MDA-SMAC protocol is still less than that of the S-MAC protocol, in larger network traffic [2123]. With increasing intervals of packet sending, energy consumption for both protocols are reduced.

Data latency comparison under the two-hop scene in MDA-SMAC protocol and S-MAC protocol is shown in Fig. 10. As can be seen from the figure, the next twohop scenario, compared to hop scenario, the transmission delay that has a certain data packet is increased. In high network traffic, compared to the S-MAC protocol, due to the MDA-SMAC protocol, using an adaptive duty cycle and adaptive back-off algorithm can reduce the impact of conflict on data transmission, so data latency is less than S-MAC protocol. With the increase of contract intervals, in both reducing delays, delays in MDA-SAMC agreement are still less than the S-MAC protocol [2426].

\section{Conclusions}

This paper studies the competition-based MAC protocol and analyzes its key technologies, related algorithms, the classic S-MAC protocols based on the competition, and analyzed its performance and shortcomings. Then, an improved S-MAC protocol is proposed with following innovations: (1) in wireless sensor networks, data collisions wasted the most energy, while the data collision probability is closely related to the number of nodes in channel competition: the more nodes competing for channel, the greater the collision probability becomes. How to reduce the node number that competes for channel in the same time becomes a vital research direction of reducing the collision probability. On this basis, we propose a new scheduling cycle: the micro-cycle, which divides a whole listening/sleep time into several small listening/sleep cycle. The number of micro-duties is determined by residual energy, with different states set

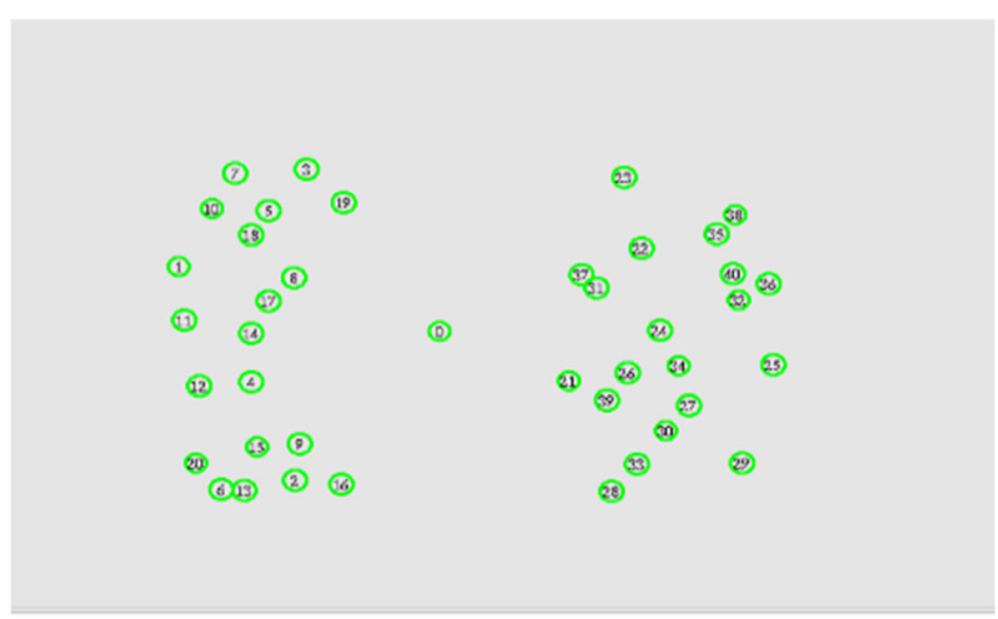

Fig. 8 The topo of two hops 


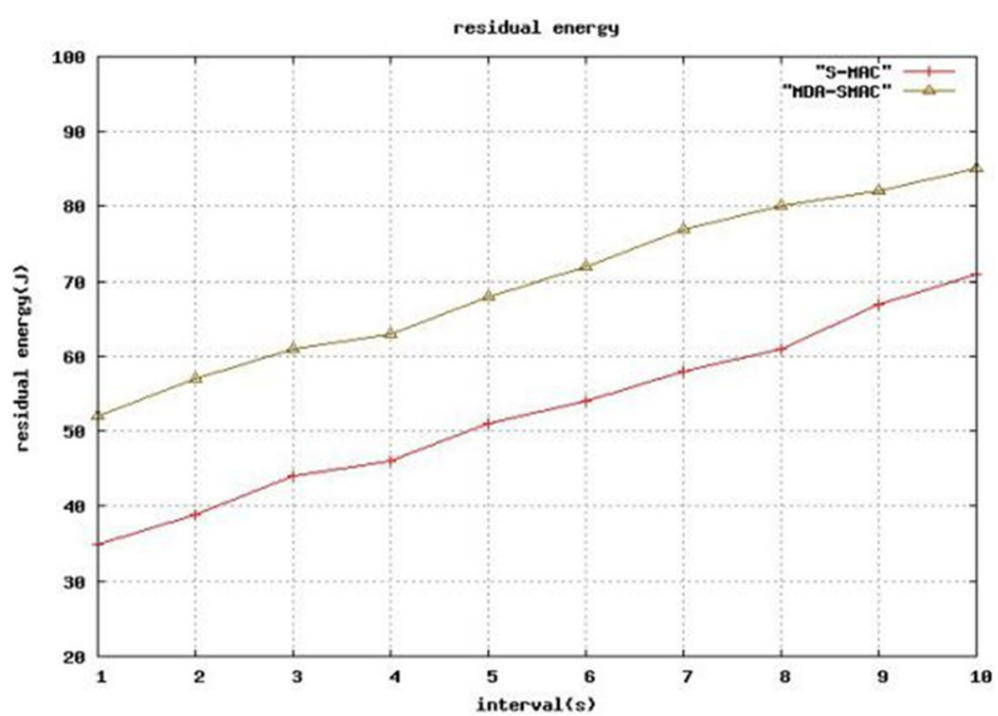

Fig. 9 The comparison of residual energy of nodes

scattering inside each micro-cycle. Channel competing level is reduced by scheduling each contention in each micro-cycle, reducing the competing level, which in turn reduces the probability of conflict and saves node energy. Meanwhile, nodes working in multiple micro-cycles transmit data in a similar TDMA manner, which decreases data latency of network. (2) To solve the problem that S-MAC protocol cannot properly adapt to the traffic dynamic change due to its fixed duty cycle in WSN, we propose a traffic selfadaptive duty cycle mechanism for micro-cycle. Duty cycle is adjusted according to its buffer queue length (the duty cycle of the micro-cycle will be multiplied if the buffer queue length is larger than the threshold value). Besides, this paper also studied the adaptive back-off algorithm: for different duty cycle, different back-off algorithms will be selected correspondingly

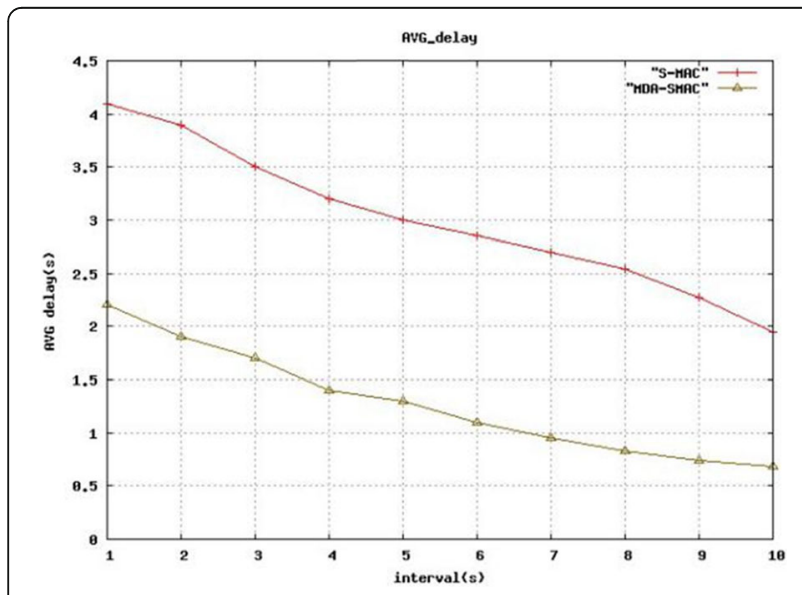

Fig. 10 The average delay of two hops based on the buffer utilization (for standard duty cycle, if the buffer utilization is less than $20 \%$, then F-BEB fast back-off algorithm will be applied for reducing data latency; for double duty cycle, CA-BEB algorithm will be used to reduce the conflict probability as much as possible and further conserves node energy.

MDA-SMAC protocol is proposed by applying microcycles, adaptive micro-cycle duty cycle mechanism and adaptive back-off algorithm in the original SMAC protocol. Comparing the simulation results for both protocols, it is obvious that MDA-SMAC protocol performs better in terms of energy consumption and delay than S-MAC, especially in high-flow state.

\section{Abbreviations}

WSN: Wireless sensor netMACMedia Access Control ProtocolMDASMACDivision-Multiple Access- Media Access Control ProtocolS-MACSensorMedia Access Control ProtocolCA-BEBConflict-Avoid-Binary exponential BackoffF-BEBFast-Binary Exponential BackoffT-MACTime-Media Access Control ProtocolTDMATime-Division-Multiple Access

\section{Authors' contributions}

All authors have contributed to this research work. All authors have read and approved the final manuscript.

\section{Funding}

This work was supported by National Key and Development Project No.2017YFC0804404

\section{Competing interest}

The authors declare that they have no competing interests.

\section{Author details}

'School of Computer Science and Technology, China University of Mining and Technology, Xuzhou, China. ${ }^{2}$ The National Joint Engineering Laboratory of Internet Applied Technology of Mines, Xuzhou, China. ${ }^{3}$ Mine Digitization Engineering Research Center of Ministry of Education of the People's Republic of China, Xuzhou, China. 
Received: 10 May 2019 Accepted: 2 January 2020

Published online: 31 January 2020

\section{References}

1. H.C. Lee, J.W. Lee, J.H. Hwang, H. Yoe, A study on energy efficient MAC protocol of wireless sensor network for ubiquitous agriculture. Lecture Notes in Computer Science 6485, 642-650 (2010)

2. Haigang Gong, Xue Zhang, Linfei Yu, Xiaomin Wang, Fasheng Yi. A study on MAC protocol for wireless sensor networks. 2009 International Conference on Frontier of Computer Science and Technology.

3. XY Liu, Y Zhu, L Kong, C Liu, Y Gu, AV Vasilakos, MY Wu, CDC: compressivedata collection for wireless sensor networks. IEEE Trans. Parallel \&Distribut. Syst. 26, 2188-2197 (2015).

4. $\mathrm{Xu}$, Donghong, Ke. Wang. An adaptive traffic MAC protocol based on correlation of nodes. Eurasip Journal on Wireless Communications \& Networking 2015.1(2015):258, 1-13.

5. D. Xu, Ke. Wang Stochastic modeling and analysis with energy optimization forwireless sensor networks. International Journal of Distributed Sensor Networks.

6. Wang K, Xu D*. Connectivity node set generation algorithm of mine WSN based on the maximum distance. International Journal of Distributed Sensor Networks,1 2016.07.14, 12(7): 1 6

7. MJ Azhar, S Kose, in Circuits and Systems (ISCAS), 2014 IEEE International Symposium On. An enhanced pulse width modulator with adaptive duty cycle and frequency control (IEEE, 2014), pp. 958-961.

8. H Rasouli, YS Kavian, HF Rashvand, ADCA: adaptive duty cycle algorithmfor energy efficient IEEE 802.15.4 beacon-enabled wireless sensornetworks. Sensors J. IEEE. 14(11), 3893-3902 (2014).

9. P. Patel, D.K. Lobiyal, A simple but effective contention aware and adaptive back-off mechanism for improving the performance of IEEE 802.11 DCF[J]. Wireless Personal Communications 83(3), 1801-1841 (2015)

10. P Shi, Y Wang, K Li, AT Chan, in Pervasive Computing and the NetworkedWorld. Cross-layer adaptive end-to-end delay control for asynchronous duty-cycle wireless sensor networks, (2014), pp. 520-531.

11. KK Sharma, M Aggarwal, N Yadav, Squaring back off based media access control for vehicular ad-hoc networks, (2014), pp. 447-455.

12. Shurman M, Al-Shua'B B, Alsaedeen M, et al. N-BEB: New backoff algorithm for IEEE 802.11 MAC protocol[C]// International Convention on Information \& Communication Technology, Electronics \& Microelectronics. 2014.

13. H.R. Hussen, C.R. Teja, T. Miao, et al., Traffic-aware cooperative binary exponential backoff algorithm for low power and lossy networks. Wireless Personal Communications 86(4), 1913-1929 (2016)

14. Bachir A, Dohler M, Watteyne T, Leung KK. MAC essentials for wireless sensor network. IEEE Communications Surveys \& Tutorials, Vol. 12.No.2, Second Quarter 2010.

15. Y. Gao, W. Cheng, H. Zhang, Z. Li, Heterogeneous statistical QoS provisioning overwireless powered sensor networks. IEEE Access 5, 79107921 (2017)

16. P.D. Diamantoulakis, K.N. Pappi, Z. Ding, G.K. Karagiannidis, Wirelesspowered communications with non-orthogonal multiple access. IEEE Trans. Wirel. Commun. 15, 8422-8436 (2016)

17. S. Zhang, N. Zhang, S. Zhou, J. Gong, Z. Niu, X. Shen, Energy-aware traffic offloading for green heterogeneous networks. IEEE J. Sel. Areas Commun. 34, 1116-1129 (2016)

18. C. Erhan, Introduction to Stocastic Processes (Prentice-Hall, Upper Saddle River, NJ, 1975)

19. N. Zhao, F.R. Yu, V.C.M. Leung, Opportunistic communications in interference alignment networks with wireless power transfer. IEEE Wirel. Commun. 22, 88-95 (2015)

20. S. Guo, F. Wang, Y. Yang, B. Xiao, Energy-efficient cooperative transmission for simultaneous wireless information and power transfer in clustered wireless sensor networks. IEEE Trans. Commun. 63, 4405-4417 (2015)

21. X. Lu, P. Wang, D. Niyato, D.I. Kim, Z. Han, Wireless networks with RF energy harvesting: a contemporary survey. IEEE Commun. Surv. Tutor. 17, 757-789 (2015)

22. Reiner Jedermann etc. Applying autonomous sensor systems in logisticscombining sensor networks, RFIDs and software agents[]]. Sensors and Actuators A:Physical. 2006,132(1): 370-375.

23. Bachir A, Dohler M, Watteyne T, Leung KK. MAC essentials for wireless sensor network. IEEE Communications Surveys \& Tutorials, Vol. 12.No.2, Second Quarter 2010.
24. M.D. Jovanovic, G.L. Djordjevic, TFMAC: Multi-channel MAC protocol for wireless sensor networks[C]. In:Proc. of 8th International Conference on Telecommunications in Modern Satellite. Cable and Broadcasting Services 26(28), 23-26 (2007)

25. Zhao Xiaohu, Hu Dongping, Li Zhi, You Peng Optimized consensus time synchronization for microseismic monitoring Journal of China University of Mining Technology, 2017, 46(5),1166-1174.

26. H. Xu, Y.G. Yang, X. Wang, M.M. Liu, H.X. Xie, C.J. Wang, Multiple kernel dimensionality reduction via ratio-trace and marginal fisher analysis, mathematical problems in engineering, Volume (2019), 8 pages

\section{Publisher's Note}

Springer Nature remains neutral with regard to jurisdictional claims in published maps and institutional affiliations.

\section{Submit your manuscript to a SpringerOpen ${ }^{\circ}$ journal and benefit from:}

- Convenient online submission

- Rigorous peer review

- Open access: articles freely available online

- High visibility within the field

- Retaining the copyright to your article

Submit your next manuscript at $\boldsymbol{\sim}$ springeropen.com 